\title{
Editorial
}

\author{
Martin Leníček
}

\section{Mathematical recalibration of total bile acids: comparing the incomparable?}

https://doi.org/10.1515/cclm-2021-0793

Keywords: bile acids; cholestasis; enzymatic assay.

To the Editor,

One of the most beneficial medical outcomes of globalization is the easiness to perform multi-center international clinical/research studies. On the other hand, we are still facing inconsistencies in and difficulties with commutability and comparability of laboratory results; as the idea of any universal analytical setup across the globe is obviously Utopian and unfeasible. To prevent the mixing of apples and oranges, thorough standardization is required, which becomes particularly ambitious if there is no reference method or reliable calibrator available. At this point, complex statistical and mathematical approaches may help to reduce variability within the results: for example, this has been done in the effort to harmonize the results of thyroid function tests [1]. In the current issue of the Journal, Corey et al. accepted the challenge of standardizing the outcomes of enzymatic assay-based determinations of total bile acids (BA) across those centers participating in the TURRIFIC randomized trial [2, 3]. Taking into consideration the differences between the individual kits of various suppliers [4], as well as the lack of accessible reference materials, they decided to apply mathematical harmonization. To prove its feasibility, the authors simulated the raw data from eight hypothetical laboratories (differing in systematic error, proportional bias, and analytical CV) and then recalibrated them by a weighted regression approach. Although the simulated input data were far from perfect, the output was surprisingly homogeneous. This gives us hope that mathematical recalibration may be a powerful tool for harmonizing data even from poorly performing laboratories. However, even pleasing harmonization should not lull us into false feeling of accuracy, as

Martin Leníček, MD, PhD, Institute of Medical Biochemistry and Laboratory Diagnostics, 1st Faculty of Medicine, Charles University, Kateřinská 32, 12108 Prague, Czech Republic, E-mail: martin.lenicek@lf1.cuni.cz. https://orcid.org/0000-00016242-3488 harmonized/standardized results are not always correct. Sometimes, mathematical recalibration cannot compensate for the systematic error that arises from the nature of the assay itself and, in fact, may affect accuracy in real

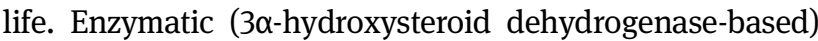
determination of total BA is a paradigmatic example. Individual BA species exhibit various reactivity towards the enzyme, leading to overall total BA underestimation [5]. As the extent of the underestimation depends on the BA spectrum, extra attention should be paid to samples, where significant changes of the spectrum are to be expected. The ongoing TURRIFIC trial [3] examines the efficacy of rifampicin over ursodeoxycholic acid (UDCA) treatment in intrahepatic cholestasis of pregnancy. Both treatment options are expected to significantly alter the BA spectrum. While UDCA administration markedly increases the proportion of UDCA (and its glyco/tauro conjugates), the action of rifampicin is more complex. As a potent agonist of the PXR receptor, it not only inhibits CYP7A1, but it also activates CYP3A4 leading to lower production of primary BA, and to the formation of hyo(deoxy)cholic acid (via induction of $6 \alpha$-hydroxylation), respectively [6, 7]. As all three "uncommon" BA exhibit intermediate or poor reactivity in enzymatic assays (about 70\% in UDCA, 45\% in hyodeoxycholic, and $25 \%$ in hyocholic acid - when compared to glycocholate [5]), the total BA concentration in the post-treatment samples is likely to be underestimated. Because the underestimation of the post-treatment samples will be more pronounced than for the pre-treatment ones, even the relative comparison may not be accurate and cannot be compensated for without assessing the BA spectrum. In conclusion, the mathematical recalibration of total BA results appears to be a viable approach, which may save the non-interchangeable data that have already been acquired. However, I believe that standardization based on either the reference materials or a pooled sample is a safer alternative, if it is logistically manageable. In studies where marked changes in the BA spectrum can be expected, an underestimation of the total BA seems inevitable; and the investigator must decide whether it is clinically relevant or not. If necessary, more sophisticated and accurate methodology (liquid chromatography with tandem mass spectrometry detection [LC-MS/MS]) is a valuable alternative. 
Research funding: This work was supported by a grant Progres Q25/LF1 from Charles University.

Author contributions: Single author article.

Competing interests: Author states no conflict of interest.

\section{References}

1. Clerico A, Ripoli A, Zucchelli GC, Plebani M. Harmonization protocols for thyroid stimulating hormone (TSH) immunoassays: different approaches based on the consensus mean value. Clin Chem Lab Med 2015;53:377-82.

2. Markus C, Coat S, Marschall H-U, Williamson C, Dixon P, Fuller M, et al. The BACH project protocol: an international multicentre total Bile Acid Comparison and Harmonisation project and sub-study of the TURRIFIC randomised trial. Clin chem Lab Med 2021;59: 1921-9.
3. Hague WM, Callaway L, Chambers J, Chappell L, Coat S, de HaanJebbink J, et al. A multi-centre, open label, randomised, parallelgroup, superiority Trial to compare the efficacy of URsodeoxycholic acid with RIFampicin in the management of women with severe early onset Intrahepatic Cholestasis of pregnancy: the TURRIFIC randomised trial. BMC Pregnancy Childbirth 2021;21:51.

4. Danese E, Salvagno GL, Negrini D, Brocco G, Montagnana M, Lippi $G$. Analytical evaluation of three enzymatic assays for measuring total bile acids in plasma using a fully-automated clinical chemistry platform. PloS One 2017;12:e0179200.

5. Zizalova K, Vecka M, Vitek L, Lenicek M. Enzymatic methods may underestimate the total serum bile acid concentration. PloS One 2020;15:e0236372.

6. Wietholtz H, Marschall HU, Sjövall J, Matern S. Stimulation of bile acid 6 alpha-hydroxylation by rifampin. J Hepatol 1996;24:713-8.

7. Staudinger JL, Goodwin B, Jones SA, Hawkins-Brown D, MacKenzie $\mathrm{KI}$, LaTour A, et al. The nuclear receptor PXR is a lithocholic acid sensor that protects against liver toxicity. Proc Natl Acad Sci U S A 2001;98:3369-74. 